\title{
EXAMPLES OF MORI DREAM SPACES WITH PICARD NUMBER TWO
}

\author{
ATSUSHI ITO
}

\begin{abstract}
In this note, we give a sufficient condition such that a projective variety with Picard number two is a Mori dream space. Using this condition, we obtain examples of Mori dream spaces with Picard number two.
\end{abstract}

\section{INTRODUCTION}

Mori dream spaces, which were introduced by $\mathrm{Hu}$ and Keel in [HK], are special varieties which have very nice properties in view of the minimal model program. In the paper, $\mathrm{Hu}$ and Keel investigated properties of Mori dream spaces, especially those related to GIT.

We recall the definition of Mori dream spaces. For a normal $\mathbb{Q}$-factorial projective variety $X$, we set

$$
N^{1}(X)_{K}:=(\operatorname{Cl}(X) / \equiv) \otimes_{\mathbb{Z}} K=(\operatorname{Pic}(X) / \equiv) \otimes_{\mathbb{Z}} K
$$

for $K=\mathbb{Q}$ or $\mathbb{R}$. We denote by $\operatorname{Eff}(X), \operatorname{Mov}(X)$, and $\operatorname{Nef}(X)$ the cones in $N^{1}(X)_{\mathbb{R}}$ generated by effective, movable, and nef divisors respectively.

Definition 1.1. By a small $\mathbb{Q}$-factorial modification (SQM) of a projective variety $X$, we mean a birational map $f: X \rightarrow X^{\prime}$ with $X^{\prime}$ projective, normal, and $\mathbb{Q}$-factorial, such that $f$ is an isomorphism in codimension one.

Definition 1.2. A normal projective variety $X$ is called a Mori Dream Space if the following hold:

i) $X$ is $\mathbb{Q}$-factorial and $\operatorname{Pic}(X)_{\mathbb{Q}}=N^{1}(X)_{\mathbb{Q}}$,

ii) $\operatorname{Nef}(X)$ is the affine hull of finitely many semiample line bundles,

iii) There is a finite collection of SQMs $f_{i}: X \rightarrow X_{i}$ such that each $X_{i}$ satisfies ii) and $\operatorname{Mov}(X)=\bigcup_{i} f_{i}^{*}\left(\operatorname{Nef}\left(X_{i}\right)\right)$.

Quasi-smooth projective toric varieties are typical examples of Mori dream spaces. In [BCHM, Corollary 1.3.2], it is shown that $\mathbb{Q}$-factorial log Fano varieties are Mori dream spaces. See [AHL, [Jo], [TVV], etc. for other examples.

By definition, a normal $\mathbb{Q}$-factorial projective variety $X$ is a Mori dream space if $\operatorname{Pic}(X)_{\mathbb{Q}} \cong \mathbb{Q}$. Then, how about the case when the Picard number is two? The following theorem gives a sufficient condition for $X$ to be a Mori dream space when the Picard number is two.

Theorem 1.3. Let $X$ be a normal $\mathbb{Q}$-factorial projective variety with Picard number 2 and $\operatorname{Pic}(X)_{\mathbb{Q}}=N^{1}(X)_{\mathbb{Q}}$. Assume that there exist nonzero effective Weil divisors $D_{1}, \ldots, D_{r}$ and $D_{1}^{\prime}, \ldots, D_{r^{\prime}}^{\prime}$ on $X$ for some $2 \leq r, r^{\prime} \leq \operatorname{dim} X$ such that

2010 Mathematics Subject Classification. 14C20, $14 \mathrm{M} 99$.

Key words and phrases. Mori dream space, small $\mathbb{Q}$-factorial modification. 
a) Cone $\left(D_{1}, \ldots, D_{r}\right) \cap \operatorname{Cone}\left(D_{1}^{\prime}, \ldots, D_{r^{\prime}}^{\prime}\right)=\{0\}$,

b) $D_{1} \cap \cdots \cap D_{r}=D_{1}^{\prime} \cap \cdots \cap D_{r^{\prime}}^{\prime}=\emptyset$.

Then $X$ is a Mori dream space.

As corollaries of Theorem 1.3, we obtain the following examples, which are special cases of Corollary 3.1. In both cases, we will construct divisors $D_{i}, D_{j}^{\prime}$ as in Theorem 1.3 by using the defining equations of $X$ or $Z$. We note that Corollary 1.4 is proved by Oguiso (at least in the case $n=3$ ) in his private note $\mathrm{Og}$.

Corollary $1.4(\underline{\mathrm{Og}})$. Let $X \in\left|\mathcal{O}_{\mathbb{P}^{1} \times \mathbb{P}^{n}}(a, b)\right|$ be a general hypersurface on $\mathbb{P}^{1} \times \mathbb{P}^{n}$ such that $a, b>0$ and $n \geq \max \{a, 3\}$. Then $X$ is a Mori dream space.

Corollary 1.5. Let $Z \subset \mathbb{P}^{N}$ be a complete intersection of general hypersurfaces of degrees $d_{1}, \ldots, d_{s}$, and let $X \rightarrow Z$ be the blow up at a general point $p \in Z$. If $Z$ is a Fano variety, i.e., if the anticanonical bundle $-K_{Z}=\mathcal{O}_{Z}\left(N+1-\sum_{i=1}^{s} d_{i}\right)$ is ample, then $X$ is a Mori dream space.

Remark 1.6. Let $X \in\left|\mathcal{O}_{\mathbb{P}^{k} \times \mathbb{P}^{n+1-k}}(a, b)\right|$ be a normal $\mathbb{Q}$-factorial hypersurface on $\mathbb{P}^{k} \times \mathbb{P}^{n+1-k}$ such that $n \geq 3$ and $a, b>0$. By the Lefschetz hyperplane theorem, it holds that $\operatorname{Pic}(X) \cong \operatorname{Pic}\left(\mathbb{P}^{k} \times \mathbb{P}^{n+1-k}\right)$.

If $2 \leq k \leq n-1, \mathcal{O}_{X}(1,0)$ and $\mathcal{O}_{X}(0,1)$ are semiample and not big. Hence $X$ is a Mori dream space since $\operatorname{Eff}(X)=\operatorname{Mov}(X)=\operatorname{Nef}(X)=\mathbb{R}_{\geq 0} \mathcal{O}_{X}(1,0)+\mathbb{R}_{\geq 0} \mathcal{O}_{X}(0,1)$. We note that this is a special case of [Jo, Corollary 2]. Since $K_{X}=\mathcal{O}_{X}(a-k-1, b-$ $(n+2-k))$, it holds that

$$
\kappa(X)=\left\{\begin{array}{cl}
-\infty & \text { for } a \leq k \text { or } b \leq n+1-k, \\
0 & \text { for } a=k+1, b=n+2-k, \\
k & \text { for } a \geq k+2, b=n+2-k, \\
n & \text { for } a \geq k+2, b \geq n+3-k,
\end{array}\right.
$$

where $\kappa(X)$ is the Kodaira dimension of $X$. Thus $\kappa(X)$ can be any number in $\{-\infty, 0,1,2, \cdots, n\}$ except 1 by choosing suitable $k, a, b$.

On the other hand, a general hypersurface $X \subset \mathbb{P}^{1} \times \mathbb{P}^{n}$ in $\left|\mathcal{O}_{\mathbb{P}^{1} \times \mathbb{P}^{n}}(3, n+1)\right|$ for $n \geq 3$ is a Mori dream space with $\kappa(X)=1$ by Corollary 1.4.

Hence for any $n \geq 3$ and $\kappa \in\{-\infty, 0,1, \cdots, n\}$, there exists an $n$-dimensional smooth Mori dream space with $\kappa(X)=\kappa$ and Picard number 2. This is shown in Og when $n=3$.

Remark 1.7. In a recent paper Ot], Ottem independently studied hypersurfaces in $\mathbb{P}^{1} \times \mathbb{P}^{n}$ more precisely by a different argument. Ottem determines for which $a, b>0$, a (very) general hypersurface $X \in\left|\mathcal{O}_{\mathbb{P}^{1} \times \mathbb{P}^{n}}(a, b)\right|$ is a Mori dream space, and describes the Cox ring of such $X$ when $X$ is a Mori dream space.

Notation. Throughout this note, we work over the complex number field $\mathbb{C}$. A divisor means a Weil divisor. For a base point free divisor $D$, we denote by $\varphi_{|D|}$ the morphism defined by the complete linear system $|D|$. We define $\operatorname{dim} \emptyset=-1$.

Acknowledgments. The author would like to express his gratitude to Professor Yujiro Kawamata for his valuable comments and advice. He is also grateful to Professor Keiji Oguiso for giving him his private note. He wishes to thank Professors Yoshinori Gongyo and Shinnosuke Okawa for useful comments and answering many questions about Mori dream spaces. 


\section{Proof of Theorem 1.3}

In this section, we prove Theorem 1.3. We will construct all the SQMs inductively by using the divisors $D_{i}, D_{j}^{\prime}$.

Definition 2.1. Let $X$ be a normal $\mathbb{Q}$-factorial projective variety with Picard number 2 and $\operatorname{Pic}(X)_{\mathbb{Q}}=N^{1}(X)_{\mathbb{Q}}$. Fix a divisor or a line bundle $A \in N^{1}(X)_{\mathbb{R}} \backslash\{0\}$. For nonzero effective divisors $D_{1}, D_{2}$ on $X$, we denote

$$
D_{1} \succeq_{A} D_{2} \quad \text { if } \quad D_{2} \in \operatorname{Cone}\left(D_{1}, A\right) .
$$

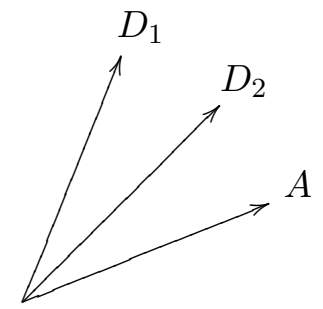

Figure 1. $D_{1} \succeq_{A} D_{2}$

Definition 2.2. Let $X$ be a normal $\mathbb{Q}$-factorial projective variety with Picard number 2 and $\operatorname{Pic}(X)_{\mathbb{Q}}=N^{1}(X)_{\mathbb{Q}}$. Let $D_{1}, \ldots, D_{k+1}$ be nonzero effective divisors on $X$ for $0 \leq k \leq \operatorname{dim} X-1$. We say that $D_{1}, \ldots, D_{k+1}$ satisfy condition (*) if $D_{1} \succeq_{A} \cdots \succeq_{A}$ $D_{k+1}$ for an ample line bundle $A$ and the following conditions 1) - 4) hold.

1) $D_{k+1}$ is semiample,

2) $D_{k+1} \notin$ Cone $\left(D_{1}, \cdots, D_{k}\right)$,

3) $D_{1} \cap \cdots \cap D_{k} \neq \emptyset$,

4) $\operatorname{dim} \varphi_{\left|m D_{k+1}\right|}\left(D_{1} \cap \cdots \cap D_{k}\right) \leq \operatorname{dim} X-k-1$ for sufficiently divisible $m>0$, where we set Cone $\left(D_{1}, \ldots, D_{k}\right)=\{0\}$ and $D_{1} \cap \cdots \cap D_{k}=X$ when $k=0$.

If $D_{1}, \ldots, D_{k+1}$ satisfy condition $(*), D_{k+1}$ is nef but not ample as we will prove in the following lemma. Hence $\mathbb{R}_{\geq 0} D_{k+1}$ is an edge of $\operatorname{Nef}(X)$.

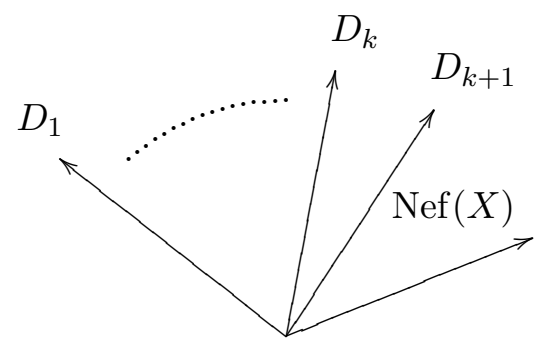

Figure 2. Condition $(*)$

Lemma 2.3. Let $X$ be a normal $\mathbb{Q}$-factorial projective variety with Picard number 2 and $\operatorname{Pic}(X)_{\mathbb{Q}}=N^{1}(X)_{\mathbb{Q}}$. Assume that divisors $D_{1}, \ldots, D_{k+1}$ on $X$ satisfy condition (*). Then $D_{k+1}$ is not ample. 
Furthermore, if $k=0, D_{k+1}$ is not big. If $k>0, D_{k+1}$ is big and $\varphi_{\left|m D_{k+1}\right|}$ is an isomorphism on $X \backslash\left(D_{1} \cap \cdots \cap D_{k}\right)$ for sufficiently divisible $m>0$.

Proof. It holds that

$$
\operatorname{dim} \varphi_{\left|m D_{k+1}\right|}\left(D_{1} \cap \cdots \cap D_{k}\right) \leq \operatorname{dim} X-k-1<\operatorname{dim} D_{1} \cap \cdots \cap D_{k},
$$

where the latter inequality follows from condition 3) and the Krull's principal ideal theorem (note that $D_{1}, \ldots, D_{k}$ are $\mathbb{Q}$-Cartier). Hence $D_{k+1}$ is not ample.

If $k=0, D_{k+1}$ is not big by condition 4) and $D_{1} \cap \cdots \cap D_{k}=X$. If $k>0$, we have $D_{k+1} \sim_{\mathbb{Q}} \alpha_{i} D_{i}+\beta_{i} A$ for some $\alpha_{i}, \beta_{i}>0$ and $1 \leq i \leq k$ by condition 2 ). Hence $D_{k+1}$ is big and $\varphi_{\left|m D_{k+1}\right|}$ is an isomorphism outside $D_{i}$ for any $1 \leq i \leq k$ since $m \beta_{i} A$ is very ample.

Lemma 2.4. Let $X$ be a normal $\mathbb{Q}$-factorial projective variety with Picard number 2 and $\operatorname{Pic}(X)_{\mathbb{Q}}=N^{1}(X)_{\mathbb{Q}}$. Let $A$ be an ample line bundle and let $D_{1} \succeq_{A} \cdots \succeq_{A} D_{r}$ be nonzero effective divisors on $X$ such that $D_{1} \cap \cdots \cap D_{r}=\emptyset$ and $2 \leq r \leq \operatorname{dim} X$. Then there exists $0 \leq k \leq r-1$ such that $D_{1}, \ldots, D_{k+1}$ satisfy condition $(*)$.

Proof. Since $D_{i} \succeq_{A} D_{r}$ for $1 \leq i \leq r$, there exist rational numbers $\alpha_{i}, \beta_{i} \geq 0$ such that $D_{r} \sim_{\mathbb{Q}} \alpha_{i} D_{i}+\beta_{i} A$. For sufficiently divisible $m, \operatorname{Bs}\left(\left|m D_{r}\right|\right) \subset D_{i}$ since $m D_{r} \sim m \alpha_{i} D_{i}+m \beta_{i} A$ and $m \beta_{i} A$ is base point free. Hence we have $\operatorname{Bs}\left(\left|m D_{r}\right|\right) \subset$ $D_{1} \cap \cdots \cap D_{r}=\emptyset$, which means $D_{r}$ is semiample. Set $Z$ be the image of $X$ by $\varphi:=\varphi_{\left|m D_{r}\right|}$ for sufficiently divisible $m$.

Let $0 \leq k_{1} \leq r-1$ be the smallest $i$ such that $\mathbb{R}_{\geq 0} D_{i+1}=\mathbb{R}_{\geq 0} D_{r}$. Since $\operatorname{Pic}(X)_{\mathbb{Q}}=$ $N^{1}(X)_{\mathbb{Q}}, D_{i}$ is $\mathbb{Q}$-linearly equivalent to a positive multiple of $D_{r}$ for each $k_{1}+1 \leq$ $i \leq r$. Hence $\varphi_{*}\left(D_{i}\right)$ is an ample divisor on $Z$ and $D_{i}=\varphi^{*} \varphi_{*}\left(D_{i}\right)$ holds for each $k_{1}+1 \leq i \leq r$.

First, we assume $D_{1} \cap \cdots \cap D_{k_{1}} \neq \emptyset$ and show that $D_{1}, \cdots, D_{k_{1}+1}$ satisfy condition (*). Since $D_{k_{1}+1}$ is $\mathbb{Q}$-linearly equivalent to a positive multiple of $D_{r}, D_{k_{1}+1}$ is semiample. By the choice of $k_{1}, D_{k_{1}+1} \notin \operatorname{Cone}\left(D_{1}, \cdots, D_{k_{1}}\right)$ holds. Hence it is enough to show the inequality $\operatorname{dim} \varphi\left(D_{1} \cap \cdots \cap D_{k_{1}}\right) \leq \operatorname{dim} X-k_{1}-1$.

Since $D_{1} \cap \ldots \cap D_{r}=\emptyset$ and $D_{k_{1}+1}, \ldots, D_{r}$ are pullbacks of divisors on $Z$, we have

$$
\varphi\left(D_{1} \cap \cdots \cap D_{k_{1}}\right) \cap \varphi_{*} D_{k_{1}+1} \cap \cdots \cap \varphi_{*} D_{r}=\emptyset .
$$

Hence it holds that

$$
\operatorname{dim} \varphi\left(D_{1} \cap \cdots \cap D_{k_{1}}\right) \leq r-k_{1}-1 \leq \operatorname{dim} X-k_{1}-1
$$

since $\varphi_{*} D_{k_{1}+1}, \ldots, \varphi_{*} D_{r}$ are ample divisors on $Z$. Hence $D_{1}, \ldots, D_{k_{1}+1}$ satisfy condition $(*)$.

Next, we assume $D_{1} \cap \cdots \cap D_{k_{1}}=\emptyset$. In this case, we replace $r$ with $k_{1}$ and repeat the above argument, and obtain $0 \leq k_{2} \leq k_{1}-1$. Since $r>k_{1}>k_{2}>\cdots \geq 0$, this process must stop and we obtain $k$ as in the statement of this lemma.

The following is the key proposition to construct SQMs inductively in the proof of Theorem 1.3 .

Proposition 2.5. Let $X$ be a normal $\mathbb{Q}$-factorial projective variety with Picard number 2 and $\operatorname{Pic}(X)_{\mathbb{Q}}=N^{1}(X)_{\mathbb{Q}}$. Let $D_{1}, \ldots, D_{k+1}$ be effective divisors on $X$ which satisfy condition $(*)$.

If $\operatorname{dim} D_{1} \cap \cdots \cap D_{k} \leq \operatorname{dim} X-2$, there exists an $S Q M X \rightarrow X^{+}$and an integer $0 \leq l \leq k-1$ such that 
- $D_{k+1}^{+}$is semiample,

- $\operatorname{Nef}\left(X^{+}\right)=\mathbb{R}_{\geq 0} D_{l+1}^{+}+\mathbb{R}_{\geq 0} D_{k+1}^{+}$,

- $D_{1}^{+}, \ldots, D_{l+1}^{+}$satisfy condition $(*)$ on $X^{+}$,

where $D_{i}^{+}$is the strict transform of $D_{i}$ on $X^{+}$for each $1 \leq i \leq k+1$. In particular, $\operatorname{Nef}\left(X^{+}\right)$is spanned by two semiample divisors $D_{l+1}^{+}, D_{k+1}^{+}$.

Proof. In Step 1, we construct $X^{\dagger}$, which is isomorphic to $X$ in codimension 1. In Step 2, we show that the normalization $X^{+}$of $X^{\dagger}$ is an SQM of $X$. In Step 3, we show the existence of $l$ as in the statement.

Step 1. By 2) in condition (*), we can write $D_{k} \sim_{\mathbb{Q}} a_{i} D_{i}+b_{i} D_{k+1}$ for some rational numbers $a_{i}>0, b_{i} \geq 0$ for each $1 \leq i \leq k$. Fix a sufficiently divisible integer $m>0$ and take the scheme-theoretic intersection

$$
C:=\bigcap_{1 \leq i \leq k} m a_{i} D_{i} \subset X
$$

Let $\phi: \widetilde{X} \rightarrow X$ be the blow up along $C$ and let $E$ be the Cartier divisor on $\widetilde{X}$ such that $\phi^{-1} I_{C}=\mathcal{O}_{\widetilde{X}}(-E)$ for the ideal sheaf $I_{C}$ of $C$.

Since $m D_{k} \sim m a_{i} D_{i}+m b_{i} D_{k+1}$ and $m b_{i} D_{k+1}$ is base point free, $\phi^{*}\left(m D_{k}\right)-E$ is base point free as well. Consider the following commutative diagram.

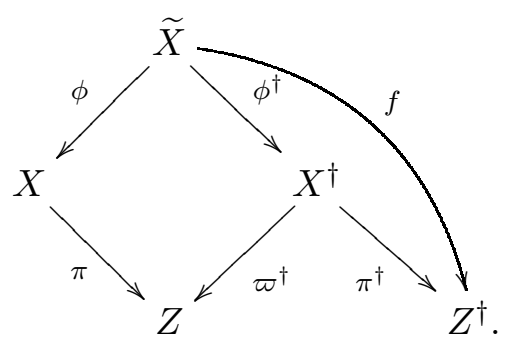

In the above diagram, $\pi:=\varphi_{\left|m D_{k+1}\right|}, f:=\varphi_{\left|\phi^{*}\left(m D_{k}\right)-E\right|}, Z=\pi(X), Z^{\dagger}=f(\tilde{X})$, $\phi^{\dagger}:=(\pi \circ \phi) \times f: \widetilde{X} \rightarrow Z \times Z^{\dagger}, X^{\dagger}=\phi^{\dagger}(\widetilde{X})$, and $\varpi^{\dagger}$ and $\pi^{\dagger}$ are the first and second projections from $X^{\dagger} \subset Z \times Z^{\dagger}$ respectively.

By Lemma 2.3, the restriction $\left.\pi\right|_{X \backslash C}: X \backslash C \rightarrow Z \backslash \pi(C)$ is an isomorphism. Since $\left.\phi\right|_{\widetilde{X} \backslash E}: \widetilde{X} \backslash E \rightarrow X \backslash C$ is also an isomorphism, the restriction of $\varpi^{\dagger}$ on $X^{\dagger} \backslash \phi^{\dagger}(E)$ is an isomorphism onto $Z \backslash \pi(C)$. Hence $X \backslash C$ and $X^{\dagger} \backslash \phi^{\dagger}(E)$ are isomorphic.

By assumption, we have $\operatorname{codim}_{X} C \geq 2$ since $C=D_{1} \cap \cdots \cap D_{k}$ as sets. To show that $X$ and $X^{\dagger}$ are isomorphic in codimension 1, it is enough to show that $\operatorname{codim}_{X^{\dagger}} \phi^{\dagger}(E) \geq 2$. By definition, there exists a natural surjection

$$
\left.\bigoplus_{i=1}^{k} \mathcal{O}_{X}\left(-m a_{i} D_{i}\right)\right|_{C} \rightarrow I_{C} / I_{C}^{2}
$$

Since $m D_{k}-m a_{i} D_{i} \sim m b_{i} D_{k+1}$, there exists an inclusion

$$
E \hookrightarrow \mathbb{P}_{C}\left(\left.\bigoplus_{i=1}^{k} \mathcal{O}_{X}\left(-m a_{i} D_{i}\right)\right|_{C}\right) \cong \mathbb{P}_{C}\left(\left.\bigoplus_{i=1}^{k} \mathcal{O}_{X}\left(m b_{i} D_{k+1}\right)\right|_{C}\right)
$$


and we have

$$
\left.\left.\mathcal{O}_{\widetilde{X}}\left(\phi^{*} m D_{k}-E\right)\right|_{E} \sim \mathcal{O}_{\mathbb{P}_{C}\left(\left.\bigoplus_{i=1}^{k} \mathcal{O}_{X}\left(m b_{i} D_{k+1}\right)\right|_{C}\right)}(1)\right|_{E}
$$

under this inclusion.

From (2.1), (2.2), and the definition of $\phi^{\dagger}$, we have

$$
\phi^{\dagger}(E) \hookrightarrow \mathbb{P}_{\pi(C)}\left(\left.\bigoplus_{i=1}^{k} \mathcal{O}_{Z}\left(m b_{i} \bar{D}_{k+1}\right)\right|_{\pi(C)}\right)
$$

for a suitable scheme structure on $\pi(C)$, where $\bar{D}_{k+1}$ is the divisor on $Z$ whose pullback on $X$ is $D_{k+1}$. Hence

$$
\begin{aligned}
\operatorname{dim} \phi^{\dagger}(E) & \leq \operatorname{dim} \mathbb{P}_{\pi(C)}\left(\left.\bigoplus_{i=1}^{k} \mathcal{O}_{Z}\left(m b_{i} \bar{D}_{k+1}\right)\right|_{\pi(C)}\right) \\
& =\operatorname{dim} \pi(C)+k-1 \leq \operatorname{dim} X^{\dagger}-2
\end{aligned}
$$

by 3) in condition $(*)$ since $\operatorname{dim} X^{\dagger}=\operatorname{dim} X$ and $C=D_{1} \cap \cdots \cap D_{k}$ as sets. Thus $\operatorname{codim}_{X^{\dagger}} \phi^{\dagger}(E) \geq 2$ holds. Hence $X$ and $X^{\dagger}$ are isomorphic in codimension 1 .

Step 2. Let $\nu: X^{+} \rightarrow X^{\dagger}$ be the normalization. Since $X^{\dagger} \backslash \phi^{\dagger}(E) \cong X \backslash C$ is normal, $\nu$ is an isomorphism in codimension 1. Hence $X \rightarrow X^{+}$is also an isomorphism in codimension 1.

To show that $X^{+}$is an SQM of $X$, it suffices to see that $X^{+}$is $\mathbb{Q}$-factorial. Let $D_{i}^{\dagger}$ be the strict transform of $D_{i}$ on $X^{\dagger}$. Since $D_{k+1}=\pi^{*} \bar{D}_{k+1}$ and $X$ and $X^{\dagger}$ are isomorphic in codimension 1 , it holds that $D_{k+1}^{\dagger}=\varpi^{\dagger *} \bar{D}_{k+1}$. In particular, $D_{k+1}^{\dagger}$ is $\mathbb{Q}$-cartier and semiample. Since $\varphi_{\left|\phi^{*}\left(m D_{k}\right)-E\right|}=f=\pi^{\dagger} \circ \phi^{\dagger}$, there exists an ample divisor $H$ on $Z^{\dagger}$ such that $\phi^{*}\left(m D_{k}\right)-E=f^{*} H=\phi^{\dagger^{*}} \pi^{\dagger^{*}} H$. Hence we have $\pi^{\dagger^{*}} H=\phi^{\dagger}{ }_{*}\left(\phi^{*}\left(m D_{k}\right)-E\right)=m D_{k}^{\dagger}$ since $E$ is contracted by $\phi^{\dagger}$ and $X \rightarrow X^{\dagger}$ is an isomorphism in codimension 1 . Thus $D_{k}^{\dagger}$ is $\mathbb{Q}$-cartier and semiample.

Let $D_{i}^{+}$be the strict transform of $D_{i}$ on $X^{+}$as in the statement of this lemma. Since $\nu: X^{+} \rightarrow X^{\dagger}$ is an isomorphism in codimension $1, D_{k}^{+}=\nu^{*} D_{k}^{\dagger}$ and $D_{k+1}^{+}=\nu^{*} D_{k+1}^{\dagger}$ hold. Hence $D_{k}^{+}$and $D_{k+1}^{+}$are $\mathbb{Q}$-cartier and semiample. Fix a prime divisor $D^{+}$on $X^{+}$. Since $D_{k}$ and $D_{k+1}$ span $N^{1}(X)_{\mathbb{Q}}=\operatorname{Pic}(X)_{\mathbb{Q}}$ as a $\mathbb{Q}$-vector space, we can write $D \sim_{\mathbb{Q}} a D_{k}+b D_{k+1}$ for some $a, b \in \mathbb{Q}$, where $D$ is the strict transform of $D^{+}$on $X$. Since $X \rightarrow X^{+}$is an isomorphism in codimension 1 , we have $D^{+} \sim_{\mathbb{Q}} a D_{k}^{+}+b D_{k+1}^{+}$, which means $D^{+}$is $\mathbb{Q}$-Cartier. Hence $X^{+}$is $\mathbb{Q}$-factorial.

Step 3. First, we show $D_{1}^{+} \cap \cdots \cap D_{k}^{+}=\emptyset$. Since $\nu\left(D_{i}^{+}\right)=D_{i}^{\dagger}$, it is enough to show $D_{1}^{\dagger} \cap \cdots \cap D_{k}^{\dagger}=\emptyset$. By Step $1, X \backslash\left(D_{1} \cap \cdots \cap D_{k}\right)=X \backslash C \cong X^{\dagger} \backslash \phi^{\dagger}(E)$. Hence we have $\left(D_{1}^{\dagger} \cap \cdots \cap D_{k}^{\dagger}\right) \backslash \phi^{\dagger}(E)=\emptyset$. Therefore it suffices to see $\left(D_{1}^{\dagger} \cap \cdots \cap D_{k}^{\dagger}\right) \cap \phi^{\dagger}(E)=\emptyset$.

For $1 \leq i \leq k$, we have an effective Cartier divisor $H_{i}$ on $\mathbb{P}_{\pi(C)}\left(\left.\bigoplus_{j=1}^{k} \mathcal{O}_{Z}\left(m b_{j} \bar{D}_{k+1}\right)\right|_{\pi(C)}\right)$ by the natural projection

$$
\bigoplus_{j=1}^{k} \mathcal{O}_{Z}\left(m b_{j} \bar{D}_{k+1}\right) \rightarrow \bigoplus_{j \neq i} \mathcal{O}_{Z}\left(m b_{j} \bar{D}_{k+1}\right)
$$


By construction, $D_{i}^{\dagger} \cap \phi^{\dagger}(E)=H_{i} \cap \phi^{\dagger}(E)$ holds as sets under the inclusion (2.3). Since $\bigcap_{i=1}^{k} H_{i}=\emptyset$, we have

$$
\bigcap_{i=1}^{k} D_{i}^{\dagger} \cap \phi^{\dagger}(E)=\bigcap_{i=1}^{k} H_{i} \cap \phi^{\dagger}(E)=\emptyset .
$$

Hence $D_{1}^{\dagger} \cap \cdots \cap D_{k}^{\dagger}$ is empty, and so is $D_{1}^{+} \cap \cdots \cap D_{k}^{+}$.

Since $D_{k}^{+}$and $D_{k+1}^{+}$are semiample by Step 2, we can take an ample line bundle $A^{+}$ on $X^{+}$such that $A^{+} \in \operatorname{Cone}\left(D_{k}^{+}, D_{k+1}^{+}\right)$. Then we have $D_{1}^{+} \succeq_{A^{+}} \cdots \succeq_{A^{+}} D_{k}^{+}$. Since $D_{1}^{+} \cap \cdots \cap D_{k}^{+}=\emptyset$, we can apply Lemma 2.4 to $D_{1}^{+}, \ldots, D_{k}^{+}$and obtain $0 \leq l \leq k-1$ such that $D_{1}^{+}, \ldots, D_{l+1}^{+}$satisfy condition $(*)$. In particular, $D_{l+1}^{+}$is semiample but not ample. Since $D_{k+1}^{+}$is semiample but not ample as well, $\operatorname{Nef}\left(X^{+}\right)$is spanned by two semiample divisors $D_{l+1}^{+}$and $D_{k+1}^{+}$, that is, $\operatorname{Nef}\left(X^{+}\right)=\mathbb{R}_{\geq 0} D_{l+1}^{+}+\mathbb{R}_{\geq 0} D_{k+1}^{+}$ holds.

Proof of Theorem 1.3. First, we show that if we relabel $D_{1}, \ldots, D_{r}$ and $D_{1}^{\prime}, \ldots, D_{r^{\prime}}^{\prime}$ if necessary, there exist integers $0 \leq k \leq r-1,0 \leq k^{\prime} \leq r^{\prime}-1$ such that $D_{1}, \ldots, D_{k+1}$ and $D_{1}^{\prime}, \ldots, D_{k^{\prime}+1}^{\prime}$ satisfy condition $(*)$ respectively. In particular, $D_{k+1}$ and $D_{k^{\prime}+1}^{\prime}$ are semiample and $\operatorname{Nef}(X)=\mathbb{R}_{\geq 0} D_{k+1}+\mathbb{R}_{\geq 0} D_{k^{\prime}+1}^{\prime}$. Hence $X$ satisfies ii) in Definition 1.2 .

Let $A$ be an ample line bundle on $X$. Assume $A \notin \operatorname{Cone}\left(D_{1}, \ldots, D_{r}\right)$. By relabeling $D_{1}, \ldots, D_{r}$ if necessary, we can apply Lemma 2.4 to $D_{1}, \ldots, D_{r}$ by the assumption b). Hence there exists $0 \leq k \leq r-1$ such that $D_{1}, \ldots, D_{k+1}$ satisfy condition (*). Since $D_{k+1}$ is nef and not contained in Cone $\left(D_{1}^{\prime}, \ldots, D_{r^{\prime}}^{\prime}\right)$, there exists an ample line bundle $A^{\prime} \notin \operatorname{Cone}\left(D_{1}^{\prime}, \ldots, D_{r^{\prime}}^{\prime}\right)$ by the assumption a). Applying Lemma 2.4 to $D_{1}^{\prime}, \ldots, D_{r^{\prime}}^{\prime}$, we obtain $0 \leq k^{\prime} \leq r^{\prime}-1$ such that $D_{1}^{\prime}, \ldots, D_{k^{\prime}+1}^{\prime}$ satisfy condition $(*)$. Since $\mathbb{R}_{\geq 0} D_{k+1} \neq \mathbb{R}_{\geq 0} D_{k^{\prime}+1}^{\prime}$, we have $\operatorname{Nef}(X)=\mathbb{R}_{\geq 0} D_{k+1}+\mathbb{R}_{\geq 0} D_{k^{\prime}+1}^{\prime}$.

If $A \in \operatorname{Cone}\left(D_{1}, \ldots, D_{r}\right)$, then $A \notin \operatorname{Cone}\left(D_{1}^{\prime}, \ldots, D_{r^{\prime}}^{\prime}\right)$ by the assumption a). Hence we can apply the same argument.

To prove that $X$ is a Mori dream space, it is enough to show that $X$ satisfies iii) in Definition 1.2. In the rest of the proof, we investigate the edges of $\operatorname{Mov}(X)$.

Case 1. First, we consider the case $\operatorname{dim} D_{1} \cap \cdots \cap D_{k}=\operatorname{dim} X$, i.e., $k=0$. In this case, $D_{1}=D_{k+1}$ is semiample and not big by Lemma 2.3. Hence $\mathbb{R}_{\geq 0} D_{1}$ is an edge of both $\operatorname{Eff}(X)$ and $\operatorname{Mov}(X)$.

Case 2. Next, we consider the case $\operatorname{dim} D_{1} \cap \cdots \cap D_{k}=\operatorname{dim} X-1$. By Lemma 2.3, $\pi:=\varphi_{\left|m D_{k+1}\right|}$ is a birational morphism for sufficiently divisible $m$. Fix a prime divisor $E$ on $X$ contained in $D_{1} \cap \cdots \cap D_{k}$. Then $E$ is contracted by $\pi$ since $\operatorname{dim} \pi\left(D_{1} \cap\right.$ $\left.\cdots \cap D_{k}\right) \leq \operatorname{dim} X-2$ by 3 ) in condition $(*)$ and $k \geq 1$. We show that $\mathbb{R}_{\geq 0} D_{k+1}$ and $\mathbb{R}_{\geq 0} E$ are edges of $\operatorname{Mov}(X)$ and $\operatorname{Eff}(X)$ respectively.

Any prime divisor on $X$ contracted by $\pi$ is $\mathbb{Q}$-linearly equivalent to $a E$ for some $a>0$ since $X$ is $\mathbb{Q}$-factorial, $N^{1}(X)_{\mathbb{Q}}=\operatorname{Pic}(X)_{\mathbb{Q}}$, and the Picard number is 2 . On the other hand, $E$ is not movable since $E$ is an exceptional divisor of $\pi$. Hence $E$ is the unique exceptional divisor of $\pi$.

Let $Z$ be the image of $X$ by $\pi$. For any prime divisor $F$ on $Z$, the strict transform $\widetilde{F}$ of $F$ on $X$ is $\mathbb{Q}$-linearly equivalent to $\alpha D_{k+1}+\beta E$ for some $\alpha, \beta \in \mathbb{Q}$. Since $\pi$ 
contracts $E$, it holds that $F=\pi_{*} \widetilde{F} \sim_{\mathbb{Q}} \pi_{*}\left(\alpha D_{k+1}+\beta E\right)=\alpha \pi_{*} D_{k+1}$. Since $\pi_{*} D_{k+1}$ is $\mathbb{Q}$-Cartier, $Z$ is $\mathbb{Q}$-factorial and $\operatorname{Pic}(Z)_{\mathbb{Q}}$ is generated by $\pi_{*} D_{k+1}$.

For a prime divisor $D \neq E$ on $X, \pi_{*}(D)$ is a prime divisor since $D$ is not contracted by $\pi$. Thus we have $\pi^{*} \pi_{*}(D) \sim_{\mathbb{Q}} b \pi^{*} \pi_{*} D_{k+1} \sim b D_{k+1}$ for some $b>0$. Since $\pi^{*} \pi_{*}(D)-D$ is effective and its support is contained in the exceptional locus of $\pi$, we have $\pi^{*} \pi_{*}(D)-D \sim_{\mathbb{Q}} a E$ for some $a \geq 0$. Thus $D \sim_{\mathbb{Q}}-a E+b D_{k+1}$. Hence $\mathbb{R}_{\geq 0} E$ is an edge of $\operatorname{Eff}(X)$ and any movable divisor is contained in $-\mathbb{R}_{\geq 0} E+\mathbb{R}_{\geq 0} D_{k+1}$. Thus $\mathbb{R}_{\geq 0} D_{k+1}$ is an edge of $\operatorname{Mov}(X)$.

Case 3. We consider the case $\operatorname{dim} D_{1} \cap \cdots \cap D_{k} \leq \operatorname{dim} X-2$. We set $X^{(0)}=X$. In this case, we can apply Proposition 2.5 to $D_{1}, \ldots, D_{k+1}$ and obtain an SQM $X^{+}$ and $D_{1}^{+}, \ldots, D_{l+1}^{+}$as in Proposition 2.5 for some $0 \leq l \leq k-1$. Set $X^{(1)}=X^{+}$, $D_{i}^{(1)}=D_{i}^{+}$, and $k_{1}=l$. If $\operatorname{dim} D_{1}^{(1)} \cap \cdots \cap D_{k_{1}}^{(1)} \geq \operatorname{dim} X^{(1)}-1$, we are reduced to Cases 1 or 2 . Hence $\mathbb{R}_{>0} D_{k_{1}+1}$ is an edge of $\operatorname{Mov}\left(X^{(1)}\right)=\operatorname{Mov}(X)$.

If $\operatorname{dim} D_{1}^{(1)} \cap \cdots \cap D_{k_{1}}^{(1)} \leq \operatorname{dim} X^{(1)}-2$, we can apply Proposition 2.5 to $D_{1}^{(1)}, \ldots, D_{k_{1}+1}^{(1)}$ and obtain another SQM $X^{(2)}$ and $D_{1}^{(2)}, \ldots, D_{k_{2}+1}^{(2)}$ for some $0 \leq k_{2} \leq k_{1}-1$. Since $k>k_{1}>k_{2}>\cdots \geq 0$ is a decreasing sequence of non-negative integers, we reach Cases 1 or 2 after repeating this process finitely many times (say, $m$ times) and obtain SQMs $X^{(1)}, \ldots, X^{(m)}$ and an edge $\mathbb{R}_{>0} D_{k_{m}+1}$ of $\operatorname{Mov}(X)$ for some $0 \leq k_{m} \leq k-1$. By Proposition 2.5. $\operatorname{Nef}\left(X^{(j)}\right)=\mathbb{R}_{\geq 0} D_{k_{j}+1}^{(j)}+\mathbb{R}_{\geq 0} D_{k_{j-1}+1}^{(j)}$ and $D_{k_{j}+1}^{(j)}, D_{k_{j-1}+1}^{(j)}$ are semiample for $1 \leq j \leq m$, where we set $k_{0}=k$.

In Cases 1 and $2, \mathbb{R}_{\geq 0} D_{k+1}$ is an edge of $\operatorname{Mov}(X)$. Following Case 3 , we set $m=0$, $k_{0}=k$, and $X^{(0)}=X$ in Cases 1 and 2. Then $\mathbb{R}_{\geq 0} D_{k_{m}+1}$ is an edge of $\operatorname{Mov}(X)$ in Cases 1, 2, 3 .

Applying the same argument to $D_{1}^{\prime}, \cdots, D_{k^{\prime}+1}^{\prime}$, we obtain SQMs $X^{\prime(0)}, \ldots, X^{\prime\left(m^{\prime}\right)}$ for some $m^{\prime} \geq 0$ (note $X=X^{(0)}=X^{\prime(0)}$ is the identity SQM) and another edge $\mathbb{R}_{\geq 0} D_{k_{m^{\prime}}^{\prime}+1}^{\prime}$ of $\operatorname{Mov}(X)$. Then $\operatorname{Mov}(X)=\mathbb{R}_{\geq 0} D_{k_{m}+1}+\mathbb{R}_{\geq 0} D_{k_{m^{\prime}}^{\prime}+1}^{\prime}$ is the union of $\operatorname{Nef}\left(X^{(j)}\right), \operatorname{Nef}\left(X^{\prime\left(j^{\prime}\right)}\right)$ for $0 \leq j \leq m, 0 \leq j^{\prime} \leq m^{\prime}$. Since the nefcone of each SQM is spanned by two semiample divisors, $X$ satisfies iii) in Definition 1.2.

\section{ExAmples}

Corollaries 1.4, 1.5 are special cases of Corollary 3.1. To show Corollary 3.1, we give divisors satisfying conditions a), b) in Theorem 1.3 explicitly. To clarify the idea, we prove Corollary 1.4 first.

Proof of Corollary 1.4. Since $\operatorname{dim} X=n \geq 3$, it holds that $\operatorname{Pic}(X) \cong \operatorname{Pic}\left(\mathbb{P}^{1} \times\right.$ $\left.\mathbb{P}^{n}\right)$ by the Lefschetz hyperplane theorem. We denote by $\mathcal{O}_{X}(k, l)$ the restriction of $\mathcal{O}_{\mathbb{P}^{1} \times \mathbb{P}^{n}}(k, l)$ on $X$.

Let $u, v$ be homogeneous coordinates on $\mathbb{P}^{1}$. Since $H^{0}\left(\mathbb{P}^{1} \times \mathbb{P}^{n}, \mathcal{O}(a, b)\right)=H^{0}\left(\mathbb{P}^{1}, \mathcal{O}(a)\right) \otimes$ $H^{0}\left(\mathbb{P}^{n}, \mathcal{O}(b)\right), X$ is the zero section of

$$
u^{a} f_{0}+u^{a-1} v f_{1}+\cdots+v^{a} f_{a}
$$

for some general $f_{i} \in H^{0}\left(\mathbb{P}^{n}, \mathcal{O}(b)\right)$. Set $W:=\left(f_{0}=\cdots=f_{a}=0\right) \subset \mathbb{P}^{n}$. Since $f_{i}$ are general, $\operatorname{dim} W=n-a-1 \geq-1$. 
Let $\bar{D}_{i} \sim \mathcal{O}_{X}(i-1, b)$ be the effective divisor on $X$ defined by

$$
\left.\left(u^{i-1} f_{0}+u^{i-2} v f_{1}+\cdots+v^{i-1} f_{i-1}\right)\right|_{X}
$$

for $1 \leq i \leq a$. Since

$$
\left.u^{a-i+1}\left(u^{i-1} f_{0}+u^{i-2} v f_{1}+\cdots+v^{i-1} f_{i-1}\right)\right|_{X}=-\left.v^{i}\left(u^{a-i} f_{i}+\cdots+v^{a-i} f_{a}\right)\right|_{X},
$$

$D_{i}:=\bar{D}_{i}-\left.\left(v^{i}=0\right)\right|_{X} \sim \mathcal{O}_{X}(-1, b)$ is an effective divisor on $X$ for each $1 \leq i \leq a$. By the definition of $D_{i}$, we have

$$
D_{1} \cap \cdots \cap D_{a}=\left(p_{2}^{*} f_{0}=\cdots=p_{2}^{*} f_{a}=0\right)=p_{2}^{-1}(W)=\mathbb{P}^{1} \times W,
$$

where $p_{2}: \mathbb{P}^{1} \times \mathbb{P}^{n} \rightarrow \mathbb{P}^{n}$ is the second projection. Choose general members $\bar{D}_{a+1}, \ldots, \bar{D}_{n}$ in $\left|\mathcal{O}_{\mathbb{P}^{n}}(1)\right|$ and set $D_{i}=\left.\left(p_{2}^{*} \bar{D}_{i}\right)\right|_{X}$ for $a+1 \leq i \leq n$. Since $W \cap \bar{D}_{a+1} \cap \cdots \cap \bar{D}_{n}=\emptyset$, we have $D_{1} \cap \cdots \cap D_{n}=\emptyset$.

If $D_{1}^{\prime}, D_{2}^{\prime}$ are general members in $\left|\mathcal{O}_{X}(1,0)\right|$, we have $D_{1}^{\prime} \cap D_{2}^{\prime}=\emptyset$. Since $D_{1}, \ldots, D_{n}$ and $D_{1}^{\prime}, D_{2}^{\prime}$ satisfy a), b) in Theorem [1.3, $X$ is a Mori dream space.

Corollary 1.4 can be generalized by a similar argument as follows.

Corollary 3.1. Let $Y$ be a smooth Mori dream space with Picard number 1, and let $A$ be a base point free line bundle on $Y$. Let $\pi: \mathbb{P}=\mathbb{P}_{Y}\left(\mathcal{O}_{Y} \oplus A\right) \rightarrow Y$ be the $\mathbb{P}^{1}$-bundle on $Y$ and let $\mathcal{O}_{\mathbb{P}}(1)$ be the tautological line bundle on $\mathbb{P}$. Let $X=D^{1} \cap \cdots \cap D^{s} \subset \mathbb{P}$ be a complete intersection on $\mathbb{P}$ of general divisors $D^{j} \in\left|\mathcal{O}_{\mathbb{P}}\left(a_{j}\right) \otimes \pi^{*} B_{j}\right|$, where $a_{1}, \ldots, a_{s}$ are positive integers and $B_{1}, \ldots, B_{s}$ are ample and base point free line bundles on $Y$. If $\operatorname{dim} X \geq \max \left\{\sum_{j=1}^{s} a_{j}, 3\right\}, X$ is a Mori dream space.

Proof. Set $n=\operatorname{dim} X$. Since $A$ is nef and $B_{j}$ is ample, $D^{j}$ is ample. Hence $\operatorname{Pic}(X) \cong$ Pic $\mathbb{P}=\mathbb{Z} \mathcal{O}_{\mathbb{P}}(1) \oplus \pi^{*}$ Pic $Y$ by the Lefschetz hyperplane theorem and $n \geq 3$.

Let $u \in H^{0}\left(\mathbb{P}, \mathcal{O}_{\mathbb{P}}(1)\right)$ and $v \in H^{0}\left(\mathbb{P}, \mathcal{O}_{\mathbb{P}}(1) \otimes \pi^{*} A^{-1}\right)$ be the sections corresponding to the first and second summands of $\mathcal{O}_{Y} \oplus A$ respectively.

Take $f^{j} \in H^{0}\left(\mathbb{P}, \mathcal{O}_{\mathbb{P}}\left(a_{j}\right) \otimes \pi^{*} B_{j}\right)$ which defines $D^{j}$. Since

$$
H^{0}\left(\mathbb{P}, \mathcal{O}_{\mathbb{P}}\left(a_{j}\right) \otimes \pi^{*} B_{j}\right) \cong H^{0}\left(Y, \operatorname{Sym}^{a_{j}}(\mathcal{O} \oplus A) \otimes B_{j}\right) \cong \bigoplus_{i=0}^{a_{j}} H^{0}\left(Y, A^{\otimes i} \otimes B_{j}\right),
$$

we can write

$$
f^{j}=u^{a_{j}} f_{0}^{j}+u^{a_{j}-1} v f_{1}^{j}+\cdots+v^{a_{j}} f_{a_{j}}^{j}
$$

for some $f_{i}^{j} \in H^{0}\left(Y, A^{\otimes i} \otimes B_{j}\right)$. Since $X$ is general, each $f_{i}^{j}$ is a general section of the base point free line bundle $A^{\otimes i} \otimes B_{j}$. For $1 \leq i \leq a_{j}$, we define a divisor $\left.D_{i}^{j} \sim \mathcal{O}_{\mathbb{P}}(-1) \otimes \pi^{*}\left(A^{\otimes i} \otimes B_{j}\right)\right|_{X}$ on $X$ to be

$$
D_{i}^{j}=\left.\left(u^{i-1} f_{0}^{j}+u^{i-2} v f_{1}^{j}+\cdots+v^{i-1} f_{i-1}^{j}=0\right)\right|_{X}-\left.\left(v^{i}=0\right)\right|_{X},
$$

which is effective as in the proof of Corollary 1.4. Similarly as in the proof of Corollary 1.4, we have

$$
\bigcap_{1 \leq i \leq a_{j}, 1 \leq j \leq s} D_{i}^{j}=\pi^{-1}\left(\bigcap_{0 \leq i \leq a_{j}, 1 \leq j \leq s}\left(f_{i}^{j}=0\right)\right) .
$$

Since $f_{i}^{j}$ are general and $\operatorname{dim} Y=n+s-1$, it holds that

$$
\operatorname{dim} \bigcap_{i, j}\left(f_{i}^{j}=0\right)=\operatorname{dim} Y-\sum_{j}\left(a_{j}+1\right)=n-\sum a_{j}-1 .
$$


Choose general hypersurfaces $\bar{D}_{1}, \ldots, \bar{D}_{n-\sum a_{j}}$ on $Y$ and set $D_{i}=\left.\left(\pi^{*} \bar{D}_{i}\right)\right|_{X}$ for $1 \leq$ $i \leq n-\sum a_{j}$. Since $\bar{D}_{1} \cap \cdots \cap \bar{D}_{n-\sum a_{j}} \cap \bigcap_{i, j}\left(f_{i}^{j}=0\right)=\emptyset$, we have

$$
D_{1} \cap \cdots \cap D_{n-\sum a_{j}} \cap \bigcap_{1 \leq i \leq a_{j}, 1 \leq j \leq s} D_{i}^{j}=\emptyset .
$$

We set $D_{1}^{\prime}=\left.\left.(u=0)\right|_{X} \sim \mathcal{O}_{\mathbb{P}}(1)\right|_{X}, D_{2}^{\prime}=\left.\left.(v=0)\right|_{X} \sim \mathcal{O}_{\mathbb{P}}(1) \otimes \pi^{*} A^{-1}\right|_{X}$. Then we have $D_{1}^{\prime} \cap D_{2}^{\prime}=\emptyset$. Since $D_{1}, \ldots, D_{n-\sum a_{j}},\left\{D_{i}^{j}\right\}_{i, j}$ and $D_{1}^{\prime}, D_{2}^{\prime}$ satisfy a), b) in Theorem $1.3, X$ is a Mori dream space.

Proof of Corollary 1.5. When $\operatorname{dim} X=2, X$ is a Del Pezzo surface. Hence $X$ is a Mori dream space.

Thus we may assume $\operatorname{dim} X=\operatorname{dim} Z \geq 3$. Set $n=N-s$. For the blow up $\mu: \widetilde{\mathbb{P}}^{n+s} \rightarrow \mathbb{P}^{n+s}$ at a general point $p \in Z, X$ is a complete intersection on $\widetilde{\mathbb{P}}^{n+s}$ of general hypersurfaces in $\left|\mu^{*} \mathcal{O}\left(d_{1}\right)-E\right|, \ldots,\left|\mu^{*} \mathcal{O}\left(d_{s}\right)-E\right|$ since $Z$ and $p$ are general. Let

$$
\pi: \widetilde{\mathbb{P}}^{n+s}=\mathbb{P}_{\mathbb{P}^{n+s-1}}(\mathcal{O} \oplus \mathcal{O}(1)) \rightarrow \mathbb{P}^{n+s-1}
$$

be the $\mathbb{P}^{1}$-bundle obtained from $\left|\mu^{*} \mathcal{O}(1)-E\right|$. Since $\mu^{*} \mathcal{O}(1)$ is the tautological bundle of $\pi$ and $\mu^{*} \mathcal{O}(1)-E=\pi^{*} \mathcal{O}_{\mathbb{P}^{n+s-1}}(1)$, we can apply Corollary 3.1 to $Y=\mathbb{P}^{n+s-1}$, $A=\mathcal{O}_{\mathbb{P}^{n+s-1}}(1), a_{j}=d_{j}-1$, and $B_{j}=\mathcal{O}_{\mathbb{P}^{n+s-1}}(1)$ if $\operatorname{dim} X=n \geq \sum\left(d_{j}-1\right)$. This condition is nothing but the ampleness of $-K_{Z}$ by the adjunction formula.

Remark 3.2. If $Z$ is not Fano, Corollary 1.5 does not hold in general. For example, $X$ is not a Mori dream space if $Z$ is a very general quartic surface in $\mathbb{P}^{3}$ and $p \in Z$ is a very general point by [AL, Proposition 6.3]. We note that Proposition 6.3 in [AL] claims that $X$ is not a Mori dream space for some $Z$ and $p$, but their proof works for very general $Z, p$.

By checking the proof of Theorem 1.3 carefully, we can explicitly describe cones in $N^{1}(X)_{\mathbb{R}}$ for Corollaries [1.4, 1.5, or 3.1. We illustrate the description by a special case of Corollary 1.5. We leave the other cases to the reader.

Example 3.3. Let $Z \subset \mathbb{P}^{n+1}$ be a general hypersurface of degree $n+1$ for $n \geq 3$. Let $\mu: X \rightarrow Z$ be the blow up at a general point $p \in Z$ and let $E$ be the exceptional divisor. We set $H=\mu^{*} \mathcal{O}_{Z}(1)$. By the proofs of Corollaries 1.5 and 3.1, we have effective divisors $D_{i} \sim i H-(i+1) E$ on $X$ for $1 \leq i \leq n$ such that $D_{1} \cap \cdots \cap D_{n}=\emptyset$. It is easy to see $D_{1}, \ldots, D_{n}$ satisfy condition $(*)$ (see the proof of Lemma 2.4), hence $\operatorname{Nef}(X)=\mathbb{R}_{\geq 0} D_{n}+\mathbb{R}_{\geq 0} H$ as in the first paragraph of the proof of Theorem 1.3. In this case, $k$ in the proof of Theorem 1.3 is $n-1$.

Since $\operatorname{dim} D_{1} \cap \cdots \cap D_{n-1}=1 \leq \operatorname{dim} X-2$, we obtain an SQM $X^{(1)}$ of $X^{(0)}:=X$ by Case 3 in the proof of Theorem 1.3. As in the proof of Theorem 1.3, we denote by $D_{i}^{(j)}$ the strict transform of $D_{i}$ on $X^{(j)}$ (note that this $D_{i}^{(j)}$ is different from $D_{i}^{j}$ in the proof of Corollary [3.1). By the proof of Proposition 2.5, $k_{1}$ in Case 3 in the proof of Theorem 1.3 is $n-2$ in this case. Hence $\operatorname{Nef}\left(X^{(1)}\right)=\mathbb{R}_{\geq 0} D_{n-1}^{(1)}+\mathbb{R}_{\geq 0} D_{n}^{(1)}$ holds. By repeating this process, we obtain an SQM $X^{(j)}$ for each $1 \leq j \leq n-2$ such that $D_{1}^{(j)}, \ldots, D_{n-j}^{(j)}$ satisfy condition $(*)$ and $\operatorname{Nef}\left(X^{(j)}\right)=\mathbb{R}_{\geq 0} D_{n-j}^{(j)}+\mathbb{R}_{\geq 0} D_{n+1-j}^{(j)}$. On $X^{(n-2)}$, we reach Case 2 in the proof of Theorem 1.3. Thus $D_{1}^{(n-2)}$ and $D_{2}^{(n-2)}$ are edges of $\operatorname{Eff}\left(X^{(n-2)}\right)=\operatorname{Eff}(X)$ and $\operatorname{Mov}\left(X^{(n-2)}\right)=\operatorname{Mov}(X)$ respectively.

From the above argument, we have the following description: 


$$
\begin{aligned}
\operatorname{Nef}(X)=\operatorname{Nef}\left(X^{(0)}\right) & =\mathbb{R}_{\geq 0} D_{n}+\mathbb{R}_{\geq 0} H, \\
\operatorname{Nef}\left(X^{(j)}\right) & =\mathbb{R}_{\geq 0} D_{n-j}+\mathbb{R}_{\geq 0} D_{n+1-j} \quad \text { for } 1 \leq j \leq n-2, \\
\operatorname{Mov}(X) & =\bigcup_{j=0}^{n-2} \operatorname{Nef}\left(X^{(j)}\right)=\mathbb{R}_{\geq 0} D_{2}+\mathbb{R}_{\geq 0} H, \\
\operatorname{Eff}(X) & =\mathbb{R}_{\geq 0} D_{1}+\mathbb{R}_{\geq 0} E .
\end{aligned}
$$

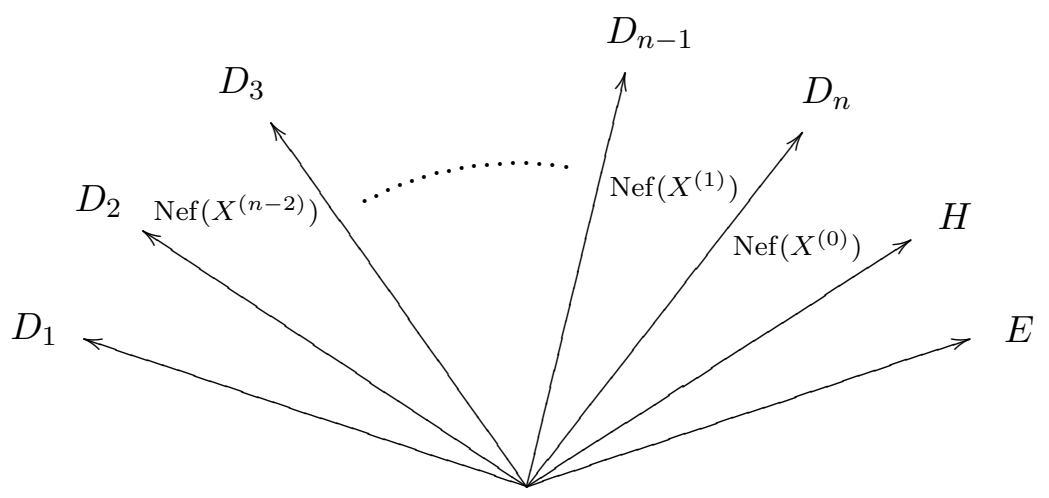

Figure 3. Cones in $N^{1}(X)$

\section{REFERENCES}

[AHL] M. Artebani, J. Hausen, and A. Laface, On Cox rings of K3 surfaces, Compos. Math. 146 (2010), no. 4, 964-998.

[AL] M. Artebani and A. Laface, Cox rings of surfaces and the anticanonical Iitaka dimension, Adv. Math. 226 (2011), no. 6, 5252-5267.

[BCHM] C. Birkar, P. Cascini, C. Hacon, and J. $\mathrm{M}^{\mathrm{c}}$ Kernan, Existence of minimal models for varieties of log general type, J. Amer. Math. Soc. 23 (2010), no. 2, 405-468.

[HK] Y. Hu and S. Keel, Mori dream spaces and GIT, Michigan Math. J. 48 (2000), 331-348.

[Jo] S-Y. Jow, A Lefschetz hyperplane theorem for Mori dream spaces, Math. Z. 268 (2011), no. 1-2, 197-209.

[Og] K. Oguiso, Mori dream hypersurfaces in the product of projective spaces, private note.

[Ot] J.C. Ottem, Birational geometry of hypersurfaces in products of projective spaces, arXiv:1305.0537.

[TVV] D. Testa, A. Várilly-Alvarado, and M. Velasco, Big rational surfaces, Math. Ann. 351 (2011), no. $1,95-107$.

Graduate School of Mathematical Sciences, The University of Tokyo, 3-8-1 Komaba, Meguro, Tokyo, 153-8914, Japan.

E-mail address: itoatsu@ms.u-tokyo.ac.jp 